\title{
Gerhard Böhme Arbeitsmarkt und Arbeitsverwaltung in der BRD
}

Die Entwicklung des Rechts der Arbeitslosenversicherung und Arbeitsförderung seit dem 2. Weltkrieg bis zur Krise 1974/75 erscheint als gradliniger Ausbau einer Arbeitsverwaltung, die den einzelnen Arbeiter kontinuierlich materiell besser stellt, ihm neue soziale Rechte einräumt und sein privates Existenzrisiko, die Verkäuflichkeit bzw. Nichtverkäuflichkeit seiner Arbeitskraft zunehmend abnimmt. Erst die Krise 1974/76 deutet mit der Reduzierung von Leistungen, der Beitragserhöhung und der Verschärfung des Zumutbarkeitsbegriffs in $\$ 103 \mathrm{AFG}^{1}$ eine entgegengesetzte Tendenz an. Diese mit dem Haushaltsstrukturgesetz vom I8. I 2. $75^{2}$ vollzogenen Einschränkungen im System der Arbeitsförderung korrespondieren mit ähnlichen Maßnahmen in anderen Sozialbereichen, die hinter den offiziellen Begründungen von der »Beschneidung des Wildwuchses « 3 einen partiellen "Abbau des Sozialstaats « ${ }^{4}$ erkennen lassen, dessen allgemeiner Kern darin liegt, soziale Dienste und Leistungen zu reduzieren bei gleichzeitiger Erhöhung der Sozialabgaben. In dem Angriff auf jene Bereiche, die bisher die Sozialstaatlichkeit der Bundesrepublik wesentlich ausmachten, erweist sich, daß der Sozialstaat und das Sozialrecht keineswegs gegen Einbrüche gesichert sind, sondern in der Krise wieder verstärkt Gegenstand der Klassenauseinandersetzungen werden.'

Um die Einschätzung der aktuellen Tendenz zu erleichtern, wird im folgenden versucht, den Ausbau und die Veränderungen des Systems der Arbeitsverwaltung nachzuzeichnen und auf die Entwicklung des Arbeitsmarktes der BRD zu beziehen. Diese läßt sich in drei Phasen einteilen ${ }^{6}:$ die 1 . Phase reicht bis ungefähr 1960 und ist gekennzeichnet durch den kontinuierlichen Abbau der Nachkriegsarbeitslosigkeit, die 2. Phase führt nach andauernder Vollbeschäftigung in die Krise 1966/67, die darauf folgende 3. Phase bringt mit zunehmender Krisenhaftigkeit des ökonomischen Prozesses und zyklischer Massenarbeitslosigkeit, in der Herausbildung einer industriellen Reservearmee die Gesetzmäßigkeiten kapitalistischer Produktion offen zur Geltung. Diese drei Phasen lassen sich auch im Recht der Arbeitslosenversicherung und Arbeitsförderung ausmachen, die gekennzeichnet sind durch den Wiederaufbau einer am Weimarer Modell orientierten Arbeitsverwaltung, die im wesentlichen auf Arbeitsvermittlung und Arbeitslosenunterstützung beschränkt war in der I. Phase, die beginnende Ausweitung arbeitsmarktpolitischer Instrumentarien und

I Vgl. dazu bereits Wacker/Paul, Der Zumutbarkeitsbegriff des Arbeitsförderungsgesetzes, KJ 1975, S. 339 .

2 BGBI. I, S. 3113.

3 Bundesarbeitsminister Arendt laut Handelsblatt vom 22. 8. 75.

4 Sozialmagazin 2/76, S. 33 ; dieser Aufsatz von Graf und Schwarz gibt einen ersten Uberblick über die Einschränkungen im Sozialbereich.

s Vgl. dazu Mückenberger, Thesen zu Funktion und Entwicklung des Sozialrechts, KJ 1976, S. 34 Iff.

6 Diese Einteilung orientiert sich an Hildebrandt, Entwicklung der Beschäftigtenstruktur und der Arbeitslosigkeit in der BRD, Prokla $19 / 20 / 21$, S. 4 I. 
punktueller Eingriffe in den Produktionsprozeß in der 2. Phase und die umfassende Ausdifferenzierung der arbeitsmarktpolitischen Ziele und Mittel im AFG in der 3. Phase, in der Qualifikation und Mobilität der Arbeitskräfte in den Mittelpunkt der Arbeitsförderung gestellt werden und diese selbst in die interventionistische Wirtschafts- und Finan politik des Staats integriert wird.

\section{Der Wiederaufbau der tradierten Arbeitsverwaltung}

Die Ausgangsbedingungen der 1. Phase sind geprägt vom Faschismus und seinen Resultaten. Das Ergebnis des 2. Weltkriegs war zwar eine politische Niederlage für die deutsche Bourgeoisie, ökonomisch aber war die Potenz des deutschen Kapitals keineswegs gebrochen: die kriegswirtschaftlichen Einschränkungen der Konkurrenz, der Zwang, den durch die Kriegsführung eingetretenen Mangel an qualifizierten Arbeitskräften durch die Einführung moderner Technologien zu überwinden und die terroristische Niederhaltung der deutschen Arbeiterklasse ermöglichten es dem deutschen Kapital, fast bis zum Kriegsende zu akkumulieren. ${ }^{7} \mathrm{Da}$ sich die Zerstörungsprozesse des Kriegs im Produktionsbereich zudem in Grenzen gehalten hatten, waren die Bedingungen für die Weiterführung der Produktion günstig. ${ }^{8}$ Demgegenüber war die deutsche Arbeiterklasse von Faschismus und Krieg ökonomisch und politisch entmachtet worden und stand dem Kapital als bloßes Arbeitslosenheer gegenüber.

In den politischen Auseinandersetzungen der Nachkriegszeit erlitt die Arbeiterbewegung neue Niederlagen. Unter der Schirmherrschaft der alliierten Militärregierungen, insbesondere der dominierenden amerikanischen Politik der "Präjudizierung durch das Verbot aller Präjudizierungen ${ }^{9}$ wurde die Restauration kapitalistischer Produktion und bürgerlicher Herrschaft betrieben. Aus hier nicht zu schildernden Gründen ${ }^{10}$ fand ein konsequenter Kampf für eine veränderte Wirtschaftsund Gesellschaftsordnung, wie sie von den Arbeiterparteien und Gewerkschaften programmatisch vertreten wurde, nicht statt. Der politische Sieg des bürgerlichen Konzepts der "sozialen Marktwirtschaft" Ludwig Erhards und die Währungsreform als einschneidendste Maßnahme der unmittelbaren Nachkriegszeit (die als Entwertung der Geldvermögen eine Enteignung der Volksmassen darstellte, während das Industriekapital und die übrigen Sacheigentümer sich dadurch entschulden konnten) bedeutete faktisch die Aussichtslosigkeit alternativer Gesellschaftskonzeptionen. Indem das Grundgesetz auf die Normierung einer Wirtschafts- und Sozialordnung verzichtete, schrieb er die bestehenden Verhältnisse zumindest faktisch fest. Die Wahlniederlage der SPD und die Ubernahme der Regierung durch den Bürgerblock unter Adenauer schloß diesen Prozeß zunächst ab und garantierte die politische Fortsetzung des restaurativen Kurses. "

Mit der Niederlage des Faschismus hatten sich auch auf dem Gebiet der Sozialversicherung Hoffnungen und Vorstellungen für einen Neubeginn verbunden. Die Sozialversicherungen waren bei Kriegsende bankrott. Ihr Vermögen bestand zu $71 \%$ aus Staatsschuldverschreibungen, mit denen sie zur Finanzierung des Kriegs

7 Vgl. Schoeller u. a., Entwicklungstendenzen des Kapitalismus in Westdeutschland (II), Prokla 16, S. ss und Schmiede, Das deutsche „Wirtschaftswunder “, in: Die Linke im Rechtsstaat, Berlin 1976, S. 107

8 Zur allgemeinen Uberschätzung des Ausmaßes der Zerstörungen vgl. die in Fn. 7 angegebene Literatur.

9 Hartwich, Sozialstaatspostulat und gesellschaftlicher status quo, Köln 1970, S. 68.

10 Vgl. die Materialien bei Huster u. a., Determinanten der westdeutschen Restauration, Frankfurt 1972; Schmidt, Die verhinderte Neuordnung, Frankfurt $1975^{\prime \prime}$.

1t Vgl. zur Nachkriegsentwicklung insgesamt Hartwich, Fn. 9 und den Sammelband: Die Linke im Rechtsstaat, Fn. 7. 
beigetragen hatten. Die internationale Diskussion um Veränderungen der Sozialversicherungssysteme schienen einen Neuanfang möglich zu machen. Im Mittelpunkt der Uberlegungen stand dabei das Modell einer Einheitsversicherung, ${ }^{12}$ in der die seit der Bismarckschen Sozialgesetzgebung selbständig organisierten Unfall-, Kranken- und Rentenversicherungen aufgehen sollten. Demgegenüber sollte die Arbeitslosenversicherung, die ein qualitativ anderes Risiko abdeckt, das unmittelbar vom Konjunkturverlauf abhängt und versicherungsmathematisch nicht erfaßtbar ist, als eigenständiger Versicherungszweig erhalten bleiben. So forderte der DGB in seinen sozialpolitischen Grundsätzen ${ }^{13}$, Arbeitslosenversicherung und Arbeitsvermittlung einer Bundesanstalt in Selbstverwaltung zu übertragen. Bei einer Massenarbeitslosigkeit, die nach Ansicht des DGB "immer durch politische Ereignisse oder Fehler der Wirtschaftspolitik verursacht" sei, sollte der Staat die zur Unterstützung der Arbeitslosen notwendigen Mittel aufbringen. Zur Bekämpfung der Arbeitslosigkeit forderte er eine staatliche Wirtschaftspolitik mit dem Ziel der Vollbeschäftigung und eine veränderte Wirtschaftsordnung. ${ }^{14}$

Auch diese Pläne für eine umfassende Sozialreform scheiterten. Die Vereinheitlichung der Sozialversicherung wurde nur in der SBZ konsequent durchgeführt, ähnliche Formen der Einheitsversicherung in der französischen Zone, Bremen und Westberlin wurden sukzessiv rückgängig gemacht. Statt dessen wurde das traditionell gegliederte, in einzelne Träger zersplitterte System der Sozialversicherung restauriert.'s Auch im Bereich der Arbeitsverwaltung und Arbeitsmarktpolitik konnten sich die Vertreter einer planenden und gestaltenden staatlichen Arbeitsmarktpolitik nicht durchsetzen. Regulativ blieb der wfreie Arbeitsmarkt «; die Arbeitsverwaltung wurde im wesentlichen auf Arbeitvermittlung und Arbeitslosenunterstützung beschränkt.

Damit war das Nachkriegsintermezzo beendet, in dem die Alliierten zunächt strenge Arbeitsmarkt- und Lohnkontrollen eingeführt hatten. Neben einem allgemeinen Lohnstop hatten sie einen Registrierungszwang für alle Erwerbstätigen und Erwerbslosen bei den deutschen Arbeitsämtern verfügt. ${ }^{16}$ Daneben wurde versucht, durch Einstellungs- und Kündigungszwangsmaßnahmen die Verteilung der Arbeitskräfte zu dirigieren. ${ }^{7}$ Diese Arbeitszwangsverwaltung wurde jedoch von allen Seiten abgelehnt. Nachdem mit Konsolidierung der Lage der Lohnstop in der amerikanischen Zone bereits gelockert worden war, hob der Wirtschaftsrat ihn danach ganz auf, ${ }^{18}$ während die Arbeitsämter sich mehr und mehr auf die Arbeits-

I 2 Material zu der Entwicklung in Großbritannien, Frankreich und Belgien bei Schieckel, Materialien zu den Gegenwartsproblemen der Sozialversicherung, Ergänzungsband, München 1948; zum Beveridge Plan für England vgl. auch Fuhrke, Staatliche Sozialpolitik, Offenbach 1976, S. 57. Die Einheitsversicherung hätte die Verallgemeinerung und Vereinheitlichung der sozialen Sicherung vorantreiben, finanziell und verwaltungsmäßig effektiver arbeiten können und, insbesondere im Bereich der Krankenversorgung ein modernes System sozialer Medizin bei Entmachtung der ärztlichen Standesorganisationen möglich gemacht. Deren Widerstand trug maßgeblich zum Scheitern der Sozialreform bei. Vgl. Fuhrke, a. a. O., m. w. N.

13 Protokoll des Gründungskongresses des DGB, Köln 1950, S. 326.

14 Wirtschaftspolitische Grundsätze des DGB, a. a. O.; vgl. auch das Referat von Agartz auf dem SPD Parteitag 1946, abgedruckt bei Huster, Fn. 10, S. 370.

is Vgl. dazu im einzelnen Tennstedt, Sozialgeschichte der Sozialversicherung, in: Handbuch der Sozialmedizin (Hg. Blomke u. a.) Bd. III, Stuttgart 1976, S. 385 (414 ff.) und Fuhrke, Fn. 12, S. $64 \mathrm{ff}$.

16 Vgl. dazu Weller, Arbeitslosigkeit und Arbeitsrecht, Stuttgart 1969, S. 151.

17 Wie bei vielen Maßnahmen am Arbeitsmarkt orientierte man sich an Regelungen aus der Zeit nach dem 1. Weltkrieg. Nach der VO über die Freimachung von Arbeitsplätzen vom 28. 3. 1919 konnten die Demobilmachungsbehörden Kündigungen verordnen, um kriegsbedingte Stellenwechsel zu revidieren. Vgl. Preller, Sozialpolitik in der Weimarer Republik, Stuttgart 1949, S. 236. Zu den entsprechenden Maßnahmen nach dem 2. Weltkrieg vgl. Weller, a. a. O.

I8 Allerdings mit erheblicher Verzögerung gegenüber der Aufhebung des Preisstops, der größtenteils bereits mit der Währungsreform entfiel. Vgl. Hartwich a. a. O., S. 102. 
vermittlung beschränkten. Die Unterstützung Erwerbsloser, nach Kriegsende eingestellt, wurde erst allmählich wieder aufgenommen. Grundlage hierfür war die Verordnung über Arbeitslosenhilfe vom 5. 9. 39. Mit dieser Verordnung hatte der Nationalsozialismus das Versicherungsprinzip in der Arbeitslosenversicherung (Beitragspflicht und Rechtsanspruch auf Unterstützung) das seit dem Inkrafttreten des Gesetzes über Arbeitsvermittlung und Arbeitslosenversicherung (AVAVG) bestanden hatte, abgeschafft und die Unterstützungszahlungen wieder an die $\mathrm{Be}$ dürftigkeit des Arbeitslosen gebunden, wodurch die Arbeitslosenunterstützung, wie in ihren Anfängen, wieder fürsorgerischen Charakter annahm. ${ }^{19}$

Im Rahmen des nie völlig außer Kraft gesetzten AVAVG begann man nun in den einzelnen Ländern, wieder eine auf dem Versicherungsprinzip aufbauende Arbeitsverwaltung zu errichten. In unterschiedlichem Ausmaß wurden Regelungen erlassen über Arbeitsvermittlung, Arbeitslosenversicherung und -fürsorge, Kurzarbeitergeld, Stillegungsvergütung und Notstandsarbeiten; die Gesetzgebungskompetenz des Bundes nach Art. 74 GG wurde erst nach und nach wahrgenommen. ${ }^{20}$ Die Zahl der Arbeitslosen, auf die sich der Aufbau der Arbeitsverwaltung zu beziehen hatte, erreichte I 950 ihren höchsten Stand mit I, 585 Mill. (10,4\%) und sank kontinuierlich bis 1960 auf 237 Tsd. (2,1\%)." Dieser anhaltende Abbau der Arbeitslosigkeit vollzog sich im Rahmen der allgemeinen Ausdehnung der abhängig Arbeitenden um ca. 5,5 Mill. ${ }^{22}$ Der ununterbrochene Wachstumsprozeß der Wirtschaft, der die Jahre des "Wirtschaftswunders" prägte, beruhte auf dem außergewöhnlichen Akkumulationsprozeß des westdeutschen Kapitals. Das relativ hohe Ausgangsniveau der Produktion und die niedrigen Löhne, deren Steigerungsraten gering blieben, ermöglichten dem Kapital, die Produktion extensiv auszudehnen und, wegen der zunächst kaum zunehmenden organischen Zusammensetzung des Kapitals, eine hohe Profitrate aufrechtzuerhalten. ${ }^{23}$

Die Staatstätigkeit bestand während dieser Phase lediglich darin, mittels Steuer- und Geldpolitik die Kapitalbildung global zu fördern und durch die Beschränkung des Staatsverbrauchs den starken Anstieg der privaten Investitionen zu begünstigen.

19 Mit der ungefähr 1936 einsetzenden Vollbeschäftigung war nicht mehr die Arbeitslosigkeit das Hauptproblem der nationalsozialistischen Arbeitsmarktpolitik, sondern die Beschaffung von Arbeitskräften. So konnte die Unterstützung Erwerbsloser auf alle Erwerbstätigen ausgedehnt werden, wurde aber an das Zurverfügungstehen für den Arbeitseinsatz gekoppelt. Vgl. insgesamt Mason, Arbeiterklasse und Volksgemeinschaft. Opladen 1975.

20 Eine Ubersicht über die einzelnen landesrechtlichen Regelungen findet sich bei Henkelmann, Das neue Recht in der Arbeitsvermittlung und Arbeitslosenversicherung, Köln 1950t.

2I Jahresdurchschnitszahlen der Arbeitslosigkeit (in \%)

$1947 \quad 595000$

$1948 \quad 603000$

$1949 \quad 1260000$

$1950 \quad 1585246 \quad(10,4)$

$1951 \quad$ I $430817 \quad(9,1)$

$1952 \quad$ I $350627 \quad(8,5)$

$1953 \quad 1251809 \quad(7,6)$

$1954 \quad 1221000 \quad(7,1)$

$1955 \quad 928308 \quad(5,2)$

$\begin{array}{lll}1956 & 761413 & (4,2) \\ 1957 & 662334 & (3,5)\end{array}$

$1958 \quad 680000 \quad(3,6)$

$1959 \quad 479926 \quad(2,5)$

$1960 \quad 237000 \quad(1,2)$

Zahlen nach Weller, a. a. O., S. 114,153 , und Bergmann u. a., Gewerkschaften in der Bundesrepublik, Frankfurt 1974, S. 346

22 Von ca. 13,6 Mill. auf ca. 19 Mill. (mit Saarland + Berlin 1960 ca. 20,2 Mill.)

23 Vgl. Schoeller u. a., a. a. O., S. 102 und die Beilage zu Handbuch I, Perspcktiven des Kapitalismus (Hg. Brandes), Frankfurt 1974 
"Der entscheidende Punkt hierbei ist, daß die Bedingungen der Kapitalakkumulation nicht mehr durch den westdeutschen Staat hergestellt werden mußten. Dadurch war es möglich, die Dinge liberak sich entwickeln zu lassen «, ${ }^{24}$ was sich in der ideologischen Verkehrung des Verhältnisses von staatlichem Handeln und Okonomie als Erfolg des neoliberalen Konzepts "sozialer Marktwirtschaft « darstellte. Bei dieser funktionierenden Komplementarität von ökonomischer Entwicklung und staatlicher Politik ${ }^{25}$ konnte sich die Arbeitsverwaltung darauf beschränken, organisatorische und rechtliche Vereinheitlichungen vorzunehmen. Da wie erwähnt das AVAVG und die Arbeitsverwaltung zunächst landesrechtlich wiederaufgebaut worden waren, kam es zu Unterschieden von Land zu Land in Art und Ausmaß der Leistungen. Die bundesweite Vereinheitlichung vollzog sich in zwei Etappen. 1952 wurde eine bundeseinheitliche Verwaltung geschaffen, die Bundesanstalt für Arbeitsvermittlung und Arbeitslosenversicherung, mit Landesarbeitsämtern und $\mathrm{Ar}$ beitsämtern als Unterbau. ${ }^{26}$ Die rechtliche Vereinheitlichung zog sich über die gesamte 2. Wahlperiode des Bundestages hin und wurde erst mit der Neuverkündung des AVAVG 1957 abgeschlossen. ${ }^{27}$

Die Verwaltung der Bundesanstalt wurde in Anlehnung an die Struktur der Reichsanstalt der Weimarer Republik aufgebaut. Die Organe der Bundesanstalt, Verwaltungsausschuß, Verwaltungsrat und Vorstand wurden drittelparitätisch aus Vertretern der Arbeitnehmer, Arbeitgeber und der öffentlichen Körperschaften gebildet. Um diese Parität hatte es scharfe Auseinandersetzungen gegeben. Die Gewerkschaften hatten sich in Verfolgung ihres sozialpolitischen Programms mit den Arbeitgeberverbänden auf die Forderung nach ausschließlicher Selbstverwaltung der Bundesanstalt durch Arbeitnehmer- und Arbeitgebervertreter geeinigt. ${ }^{28}$ Demgegenüber bestanden Bundesregierung und Bundestagsmehrheit auf der drittelparitätischen Beteiligung der öffentlichen Körperschaften. Nachdem ein erster Gesetzentwurf dieses Inhalts gescheitert war, wurde ein zweiter, leicht modifizierter Entwurf verabschiedet, der die Drittelparität festschrieb. Nur für die Verwaltung der Arbeitslosenversicherung wurde die Alleinzuständigkeit von Gewerkschafts- und Arbeitsgebervertretern festgelegt.29 Auch bei den Aufgaben der Bundesanstalt orientierte man sich an der Arbeitsverwaltung der Weimarer Zeit. Im neuen AVAVG wurden im wesentlichen die beiden »klassischen "Bereiche des Arbeitsverwaltungsrechts restauriert. Zunächst ist dies die Arbeitsvermittlung inklusive Berufsberatung und Lehrstellenvermittlung. Das der Bundesanstalt für diese Tätigkeiten eingeräumte Monopol schützt einerseits den Arbeitsmarkt vor privaten, nur an Gewinn orientierten Stellenvermittlern, andererseits gliedert es die Vermittlung von

24 Schoeller u. a., a. a. O., S. 102.

2s Dies soll nicht heißen, daß ökonomische Notwendigkeiten sich umstandslos in staatliches Handeln umgesetzt hätten, sondern nur, daß die ökonomische Entwicklung der reibungslosen Akkumulation unter exzeptionellen Bedingungen den "Erfolg" der neoliberalen Wirtschaftspolitik erst ermöglichte, wie umgekehrt diese Politik, "die keine war" (Ehrenberg), den flotten Gang der Akkumulation nicht störte.

26 Gesetz über die Errichtung einer Bundesanstalt für Arbeitsvermittlung und Arbeitslosenversicherung vom 10. 3. 1952, BGBI. I, S. 123.

27 AVAVG vom 3. 4. 1957, BGBl. I, S. 322.

$28 \mathrm{Vg}$. Hattenheimer Entschließungen, RdA 1950, S. 63 (68); Bundesvorstand des DGB (Hg), Dokumente über die Errichtung der Bundesanstalt für Arbeitsvermittlung und Arbeitslosenversicherung, Düsseldorf 1952

29 Die Beteiligung der öffentlichen Körperschaften an der Selbstverwaltung in der Arbeitslosenversicherung geht auf die öffentlichen Arbeitsnachweise zurück, deren Träger die Gemeinden waren, vgl. auch Fn. 30. Die Beibehaltung bzw. Wiedereinführung der Drittelparität in der Bundesanstalt wurde von der Bundesregierung und der Parlamentsmehrheit damit begründet, daß die Bundesanstalt weitgehend Hoheitsaulgaben zu erfüllen hätte. $V_{\text {gl I }}$ zur parlamentarischen Geschichte des Gesetzes Draeger u. a., AVAVG Kommentar, Stuttgart 1961, S. 15. 
Angebot und Nachfrage am Arbeitsmarkt aus der unmittelbaren Auseinanderset-

zung zwischen Lohnarbeit und Kapital aus. ${ }^{36}$

An die Arbeitsvermittlung gekoppelt ist die Arbeitslosenversicherung. Ein Arbeitsloser hat einen Rechtsanspruch auf Arbeitslosengeld, sofern er die Anwartschaftszeit beitragspflichtiger Beschäftigung erfüllt hat, der Arbeitsvermittlung zur Verfügung steht, sich arbeit'os gemeldet und Arbeitslosengeld beantragt hat. ${ }^{31}$ Die Höhe des Arbeitslosengeldes war nach dem AVAVG so gestaffelt, daß im Durchschnitt $55 \%$ des letzten Nettolohns gezahlt wurden. ${ }^{32}$

Besteht kein Anspruch auf Arbeitslosengeld, weil er bereits abgelaufen oder die Anwartschaftszeit nicht erfüllt ist, kann die Arbeitslosenhilfe eingreifen. Zusätzliche Voraussetzung ist allerdings die Bedürftigkeit des Antragstellers; ebenso wie die Sozialhilfe steht die Arbeitslosenhilfe unter dem Grundsatz der Subsidiarität. ${ }^{33}$

Diese prinzipielle Úbereinstimmung im Subsidiaritätsgrundsatz darf jedoch den Unterschied nicht verwischen: der Bezug von Arbeitslosenhilfe ist an die ehemalige bzw. potentielle Lohnarbeiterqualität gebunden, im Gegensatz zur Sozialhilfe als "Hilfe zum Lebensunterhalt" oder "Hilfe in besonderen Lebenslagen" ist sie als Lohnersatzleistung konzipiert. Sie berechnet sich, wie das Arbeitslosengeld, nach dem ehemaligen bzw. ortsüblichen Entgelt des Arbeitslosen. Diese Lohnbezogenheit nimmt keine Rücksicht darauf, ob Hilfsbedürftigkeit im Sinne der Sozialhilfe vorliegt, noch ob sie eventuell behoben wird. ${ }^{34}$ Reicht die Arbeitslosenhilfe nicht aus, so muß gegebenenfalls die Sozialhilfe als "Ausfallbürgschaft «35 einspringen. Die Arbeitslosenhilfe ${ }^{36}$ als Z wischenstufe zwischen dem Anspruch auf Arbeitslosengeld und der Sozialhilfe war die erste Unterstützungsform für Arbeitslose: sie hob den status des Arbeitslosen aus der tatsächlichen und rechtlichen Diskriminierung des der Armenpflege Unterworfenen heraus. ${ }^{37}$ Auch nach Einführung der Arbeitslosenversicherung behielt sie die Funktion, den Arbeitslosen vor dem Absinken in die Klientel der Sozialämter zu bewahren und durch seine Verwaltung beim Arbeitsamt seine Arbeitsbereitschaft zu erhalten.

Die Koppelung zwischen Arbeitsvermittlung und Unterstützungszahlungen besteht darin, daß die Vermittlung in Arbeit den Lohnersatzleistungen vorgeht. Dabei mußte nach $\$ 76$ AVAVG nahezu jede Arbeit angenommen werden, andernfalls tritt eine Sperre der Bezüge ein. ${ }^{38}$ Diese Sanktionsmöglichkeit garantiert, daß die Stufenfolge Vermittlung - Arbeitslosengeld - Arbeitslosenhilfe eingehalten wird. ${ }^{39}$ Mit diesen Regelungen des AVAVG war ein System der Arbeitsverwaltung rekonstruiert, wie es bereits 1927 mit der erstmaligen Verabschiedung des AVAVG errichtet worden war, in einer Situation schwerer ökonomischer Krisen und scharfer Klassenkämpfe. Damals war es - weil es dem Versicherungsprinzip entsprechend einen Anspruch auf Unterstützung gewährte und soziale Selbstverwaltung einräum-

30 Sowohl Gewerkschaften wie Arbeitgeber hatren in den Anfangsjahren des Arbeitsnachweises versucht, mitrels der Organisation eigener Nachweise die Kontrolle des Arbeitsmarkts zu erzwingen. Diese Versuche scheiterten, es entstanden auf kommunaler Ebene paritätische Nachweise, die den Grundsatz der Neutralität der Vermittlung entwickelten, der über das Arbeitsnachweisgesetz von 1922 ins AVAVG einging.

31 Heute $\$ 100$ AFG.

32 Arbeitslosengeld und -hilfe bestanden aus einem Hauptbetrag und Familienzuschlägen; nach dem AFG sind die Sätze inzwischen auf $68 \%$ bzw. $58 \%$ des letzten Nettoentgelts angehoben.

$33 \mathrm{Vgl}$. dazu Barabas/Sachse, BSHG: Sozialstaatliche Versorgung oder Armenpolizei, KJ 1976, S. 359.

34 Vgl. Draeger, u. a., a. a. O., S. 8 r.

35 Barabas/Sachse, a. a. O., S. 361.

36 Früher: Arbeits/Erwerbslosenfürsorge, Krisenfürsorge.

$37 \mathrm{Vgl}$. dazu Weller, a. a. O., S. 37.

38 Im AFG von 1969 wurde diese Verpflichtung auf die Annahme zumutbarer Arbeit beschränkt, $\$ 103$ AFG.

$39 \$ 37$ AVAVG; nach $₫ S$ AFG geht jetzt auch die Vermittlung in Berufsbildungsmaßnahmen vor. 
te - von einem Teil der Arbeiterbewegung interpretiert worden als Schritt auf dem Weg zur Wirtschaftsdemokratie..$^{\circ}$ In der Festschrift des Reichsarbeitministeriums war es sogar gefeiert worden als »ein gut Stück , Lösung der sozialen Frage auf dem Boden der gegenwärtigen Wirtschaftsordnung «. ${ }^{41}$ Die Einschätzungen verloren allerdings bald ihren Boden: der ökonomische und soziale Bankrott im Gefolge der Weltwirtschaftskrise, die politische Abdankung der SPD nach dem Bruch der großen Koalition 1930 und die Machtübernahme zunächst der Brüning, Papen, Schleicher, sodann der Regierung Hitler zerschlugen diese Struktur der Arbeitsverwaltung in kürzester Zeit, soweit dies die Verwalter der leeren Kassen der Reichsanstalt für Arbeitsvermittlung und Arbeitslosenversicherung und des Reichs nicht bereits selbst getan hatten..$^{42}$

Unter den gänzlich anderen Umständen der ökonomisch expandierenden und politisch befriedeten Bundesrepublik, in der die "soziale Frage « sich nicht mehr zu stellen schien, wurde der Frage der Arbeitslosenversicherung gar keine so große Bedeutung beigemessen. Das sich gegenseitig stabilisierende Verhältnis von prosperierender Kapitalentwicklung und neoliberaler staatlicher Wirtschaftspolitik hatte einen allgemeinen Anstieg des Lebensstandards zur Folge. Sozialpolitik konnte sich darauf beschränken, diejenigen sozialen Schäden zu regulieren, die als Folgen des Kriegs und seiner politischen und geographischen Resultate auftraten, und jene Personen sozial zu sichern, die weder arbeiten noch sich aus eigener Kraft erhalten konnten. Damit wurde der Sozialstaatsgrundsatz des Grundgesetzes reduziert auf die im Prinzip subsidiäre soziale Absicherung des nicht mehr der privaten Reproduktion fähigen Individuums. Nur als letztes Mittel sollten Leistungen der Allgemeinheit zum Unterhalt der Menschen eingesetzt werden. ${ }^{43}$

Der allgemeinen Konzeption staatsfreier Okonomie nach Marktgesetzten und privater Reproduktion der Einzelnen war die Arbeitsverwaltung bruchlos eingepaßt. Sie enthielt sich des Eingriffs in den Produktionsbereich ${ }^{44}$ und beschränkte sich auf die Vermittlung von Angebot und Nachfrage am Arbeitsmarkt und die Unterstützung nicht vermittelbarer Arbeitskräfte. Die Beiträge zur Arbeitslosenversicherung konnten sukzessiv gesenkt werden ${ }^{45}$, die vom Bund nach Art. 120 GG zu tragenden Kosten der Arbeitslosenhilfe gingen ebenfalls zurück.

\section{Ansätze interventionistischer Arbeitsmarktpolitik}

Die systematische Beschränkung der Arbeitsverwaltung auf Vermittlung und Unterstützungszahlungen wird mit Beginn der Vollbeschäftigung relativiert. In die Umbruchsphase der ökonomischen Entwicklung und des Arbeitsmarkts - Ende des Abbaus der Arbeitslosigkeit und Konsolidierung der Arbeitslosenzahlen auf niedrigem Niveau - fällt eine Erweiterung des AVAVG, die eine neue Tendenz anzeigt: die beginnende staatliche Einwirkung auf den Produktionsbereich. Mit dem Gesetz

40 Naphtali u. a., Wirtschaftsdemokratie, 1928. Nachdruck Frankfurt 1968, S. I 59.

41 Deutsche Sozialpolitik 191 8-1928, Erinnerungsschrift des Reichsarbeitsministeriums, Berlin 1929, S. 9.

42 Vgl. dazu Preller a. a. O., S. 4 I 8; Timm, Die deutsche Sozialpolitik und der Bruch der großen Koalition im März 1930, Düsseldorf 1952 .

43 Im Entwurf zum AVAVG, Bt. Drs. [1/1274, S. 89 f. heißt es exemplarisch: "Lohnersatz aus öffentlichen Mitteln ist aber nur gerechtfertigt, soweit der Arbeitslose nicht über eigene Mittel oder Ansprüche zum Ersatz des Lohnausfalls verfügt ... Mit dieser Regelung wird dem gesunden Grundsatz Rechnung getragen, daß die Allgemeinheit für den einzelnen erst eintreten soll, wenn dessen eigene wirtschaftliche Kraft oder die der Familiengemeinschaft nicht ausreichen."

44 Ausnahme war das 200 Mill. DM Programm zur Arbeitsbeschaffung im Jahr i951. Vgl. dazu den Entwurf, Fn. 43.

4s Der Beitragssatz wurde von $6,5 \% 1949$ bis auf $2 \%$ ab 1957 gesenkt. 
über die Förderung der ganzjährigen Beschäftigung in der Bauwirtschaft vom 7. 12. $59^{46}$ beginnt die "Förderung " von Produktion und Beschäftigung in bestimmten Sektoren der Okonomie durch gezielte Maßnahmen der Bundesanstalt.

Innerhalb der Unterstützungszahlungen der Bundesanstalt machten die Aufwendungen für arbeitslose Bauarbeiter Ende der soer Jahre mehr als 50\% aus. ${ }^{47}$ Diese überproportionale Belastung der Finanzen der Bundesanstalt durch die Baubranche hatte ihren Grund in der saisonalen Verteilung der Bautätigkeit. Weder waren die Bauunternehmer in ihrer Ausrüstung auf das Bauen im Winter eingestellt, noch vergaben die Bauherren Aufträge für diese Jahreszeit, was zur regelmäßigen saisonalen Brachlegung von Kapital und Arbeitskräften führte..$^{8}$

Auf der Basis der expandierenden Baukonjunktur hatten die Tarifparteien zwar bereits durch differenzierte Tarifverträge den Lohnausfall teilweise gemindert, ${ }^{49}$ dies allerdings auf Kosten der Arbeitslosenversicherung. ${ }^{50}$ Inzwischen waren jedoch die technologischen Voraussetzungen für den verstärkten Winterbau gegeben. Die Interessen der Bauunternehmer an der ganzjährigen Nutzung ihres Kapitals und der Bauarbeiter an der Erhaltung ihrer Arbeitsplätze und gleichbleibendem Einkommen auch im Winter deckten sich mit denen der Arbeitslosenversicherung an der Reduzierung der Auwendungen für die Baubranche. Diese Interessenkongruenz führte zu einer "gemeinsamen Aktion von Tarifvertragsparteien und Gesetzgeber «s" mit dem Resultat der gesetzlichen Förderung des Winterbaus. Es wurde bestimmt, $\mathrm{da} ß$ aus Mitteln der Bundesanstalt Bauherren, Bauunternehmern und Bauarbeitern Darlehen und Zuschüsse bei zusätzlicher Winterbautätigkeit zur Verfügung gestellt werden konnten. ${ }^{52}$ Zusätzlich wurde die Zahlung von Schlechtwettergeld eingeführt, die zu Verbesserungen der Einkommenssituation der Bauarbeiter führte. ${ }^{33}$

Gleichzeitig mit diesem Ergänzungsgesetz wurde ein zweiter Absatz in den $\mathbb{I}_{I}$ AVAVG eingeführt, der es der Bundesregierung gestattete, durch Rechtsverordnung mit Zustimmung des Bundesrats der Bundesanstalt zusätzliche Aufgaben zu übertragen, für deren Kosten der Bund aufzukommen hatte. Diese Vorschrift ermöglichte es, auch kurzfristig, spezielle unterstützende Eingriffe am Arbeitsmarkt vorzunehmen. Die wichtigsten Anwendungsbereiche waren in den nächsten Jahren die Berlinförderung nach dem Bau der Mauer, die Gewährung von Anpassungsbeihilfen an von der Krise des Steinkohlebergbaus betroffene Arbeitskräfte und die allgemeine Förderung der beruflichen Fortbildung aus Bundesmitteln. ${ }^{54}$

46 Gesetz über die Förderung der ganzjährigen Beschäfrigung in der Bauwirtschaft und weitere Änderungen des AVAVG, BGBI. I, S. 7os.

$47 \mathrm{Vgl}$. Draeger u. a., a. a. O., S. 58. Die Saisonarbeitslosigkeit stellte schon immer ein zentrales Problem der Arbeitslosenversicherung dar und verursachte der Weimarer Reichsanstalt immense Finanzierungsprobleme; vgl. dazu Preller, a. a. O., S. 363 ff.

$48 \mathrm{Vgl}$. das Memorandum der Bundesregierung, Bundesarbeitsblatt (BABl.) 1958, S. 673 .

49 Dies war zu einem großen Teil das Ergebnis der ausgeprägt *kooperativen * Politik der IG Bau-SteineErden, die unter allen DGB Gewerkschaften das Gesellschaftssystem der BRD am weitestgehendsten akzeptierte und unter ihrem Vorsitzenden Georg Leber in ihrer Politik darauf abzielte, "gemeinsam mit den Unternehmern sozial- und wirtschaftspolitische Lösungen zu suchen, die die sozialen Interessen der Lohnabhängigen an der Erhaltung ihrer Arbeitsplätze und der Verbesserung ihrer Lebens- und Arbeitsbedingungen berücksichtigen wie die Leistungsfähigkeit des von ihnen vertretenen Wirtschaftsbereiches steigernu. Bergmann u. a., a. a. O., S. ${ }_{4} 8$.

so Einzelheiten bei Weller, a. a. O., S. 201.

si A. a. O., S. 204.

\$2 Die tatsächlichen Erfolge blieben allerdings gering, ein Einfluß auf die Winterbautätigkeit war kaum nachweisbar. Die Subventionierung der Bauherren bis 1964 betrug 165 Mill. DM, die Zahlungen an Bauunternehmer und -arbeiter waren weitaus geringer. Der Effekt der Maßnahmen bestand somit weitgehend nur in einer Kostenentlastung der Bauherren; vgl. dazu Kranz, BABl. 1964, S. 753 und Weller, a. a. O.

$53 \mathrm{Vgl}$. Leber, Unser Weg, Referat auf dem 6. ordentlichen Gewerkschaftstag der IG Bau-Steine-Erden 1963 . Sonderdruck Ffm. o. J., S. $40 \mathrm{ff}$.

\$4 14., 16. und 18. Durchführungsverordnung zum AVAVG vom 30. 1., 13.4. und 4.6.62. 
Weitere Maßnahmen schienen bei der anhaltenden Vollbeschäftigung der nächsten Jahre nicht notwendig. Die Zahl der Arbeitslosen lag zwischen 237 Tsd. 1960 und 161 Tsd. 1966"s, die der Kurzarbeiter blieb während des gesamten Zeitraums unter 10 Tsd., auch das Ende des 3. Nachkriegszyklus beeinträchtigte diesen Zustand nur geringfügig. ${ }^{6}$ Parallel dazu verlangsamte sich die Ausdehnung der Beschäftigung. Hatte die Zahl der abhängig Beschäftigten zwischen r950 und 1960 noch um 5,5 Mill. zugenommen, so stieg sie von 1960 bis 1966 nur noch um I,5 Mill. von 20,25 auf 21,76 Mill. 57 Diese Differenz deutet bereits an, daß sich hinter der Kontinuität von ökonomischem Wachstum und Vollbeschäftigung wesentliche Veränderungen im Gang der Kapitalakkumulation vollzogen, die zu Verschiebungen der Arbeitsmarktstruktur und als deren Folge zu veränderten Anforderungen an die Arbeitsverwaltung führten. Mit dem Erreichen der "Beschäftigungsschranke" um die Wende der soer/6oer Jahre wurde das Kapital gezwungen, neue Strategien der Mehrwertproduktion anzuwenden. Die in den soer Jahren dominierende Ausweitung der Produktion auf gleichbleibender technologischer Stufenleiter fand an dem Mangel an Arbeitskräften und der damit einhergehenden Verbesserung der Position der Lohnarbeit in den Lohnauseinandersetzungen ihre Grenze. Dominierend wurde nunmehr die Produktion des relativen Mehrwerts durch die Anwendung neuer technologischer Verfahren im Arbeitsprozeß; Rationalisierung und Automatisierung ergänzten und ersetzten zunehmend die einfache Erweiterung der Produktion. .8 $^{8}$

Solche Veränderungen in der technologischen Struktur des Arbeitsprozesses haben Konsequenzen in der Profitabilität des Kapitals. Der Zusammenhang kann hier nur als Interpretationsrahmen skizziert, aber nicht begründet werden. Die steigende organische Zusammensetzung des Kapitals, Resultat des höheren Anteils des konstanten zuungunsten des variablen Kapitalteils, führt - wenn keine Kompensation durch entgegenwirkende Faktoren stattfindet - zum Sinken der Profitrate des Kapitals, die sich ja auf das insgesamt vorgeschossene Kapital berechnet. 59 Der Prozeß schlug sich in der Krise 1966/67 nieder. Der Charakter kapitalistischer Produktion als Krisenzusammenhang trat damit wieder deutlich hervor.

Die technologischen wie wertmäßigen Veränderungen im Verhältnis von Produktionsmitteln und lebendiger Arbeitskraft hatten Auswirkungen auf Zahl und Qualifikationen der in den einzelnen Branchen nachgefragten Arbeitskräfte. Durch die Veränderungen der Arbeitsprozesse wurden neue Qualifikationen notwendig, es entstanden neue Berufszweige, während andere Qualifikationen überflüssig und wertlos wurden. Auch im Verhältnis der verschiedenen Branchen untereinander verschoben sich die Beschäftigungsmöglichkeiten. ${ }^{60}$ Expandierenden Branchen standen schrumpfende gegenüber, in denen die Beschäftigtenzahlen zurückgingen.

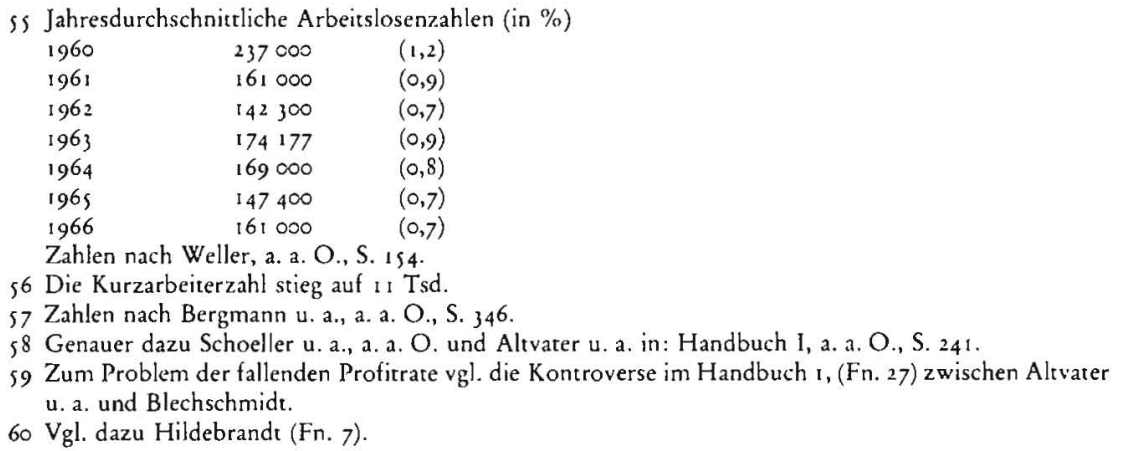


Symptomatisch dafür ist der Bergbau. Von 1958 bis Mitte 1966 verringerte sich die

Zahl der Arbeitskräfte in dieser Sparte von 694 auf 492 Tsd.6'

Dieser Beschäftigungsabbau, Resultat des Rationalisierungsprozesses im Bergbau und des Vordringens neuer Energieträger, führte zu struktureller Arbeitslosigkeit, die von den davon betroffenen bzw. bedrohten Arbeitskräften individuell nicht mehr bewältigt werden konnte. Da auf der anderen Seite nach wie vor Nachfrage nach Arbeitskräften bestand, allerdings in anderen Branchen bzw. in anderen Regionen, wurden staatliche Maßnahmen im Interesse sowohl der Arbeitskräfte wie des Kapitals notwendig.

Aus den Mitteln der Montanunion, des Bundes, der knappschaftlichen Rentenversicherung und der Arbeitslosenversicherung wurden Lohnausgleichszahlungen bei Berufswechsel, Umschulungsbeihilfen, Trennungsbeihilfen und Abfindungen gezahlt, neben regionalen Maßnahmen zur Ansiedlung neuer Industrien in den betroffenen Regionen. ${ }^{62}$ Die Entwicklung im Bergbaubereich war indes nur augenfälligster Ausdruck eines sich insgesamt verändernden Verhältnisses von Okonomie und staatlicher Arbeitsmarktpolitik. ${ }^{63}$

In allen Branchen vollzogen sich technologische Veränderungen, das Gewicht der Branchen untereinander verschob sich. Beides machte generelle Qualifikationsanpassungen der Arbeitskräfte und die Stärkung ihrer Mobilität zur Erhaltung der Flüssigkeit des Arbeitskräfteangebots nötig.

Obwohl der Großteil dieser von den Arbeitskräften verlangten Anpassungsmaßnahmen, Berufswechsel, Fortbildung etc. zunächst privat bewältigt wurde, stellte die veränderte Situation auch die Arbeitsverwaltung vor neue Anforderungen. Nicht mehr die Verwaltung von Arbeitslosigkeit stellte sich als zentrales Problem dar, sondern die Versorgung des Arbeitsmarkts mit Arbeitskräften und deren Anpassung an die veränderten Bedürfnisse der Produktion.

Diesen Anforderungen versuchte die Arbeitsverwaltung durch eine langsame Schwerpunktverschiebung ihrer Tätigkeit zu entsprechen. Die Berufsberatung bei den Arbeitsämtern wurde systematisch ausgebaut. Unter den finanziellen Leistungen der Bundesanstalt nahmen diejenigen nach Abschnitt IV des AVAVG, Maßnahmen zur Verhütung und Beendigung von Arbeitslosigkeit, ständig zu. ${ }^{64}$ Allerdings waren die Maßnahmen durch ihren subsidiären Charakter in ihrer Reichweite beschränkt; zudem war das allgemeine System der Aus- und Fortbildungsbeihilfen durch die Vielzahl der unterschiedlichen Träger zersplittert und unübersichtlich. ${ }^{65}$

Alle Maßnahmen zur Qualifikations- und Mobilitätsförderung konnten jedoch das Arbeitskräftepotential insgesamt nicht vergrößern. Insbesondere in jenen Produktionsbereichen, in denen un- bzw. angelernte Arbeitskräfte nachgefragt wurden, konnte der Bedarf nicht befriedigt werden. Die Bundesanstalt begann in dieser Situation mit dem systematischen Ausbau der Anwerbung und Vermittlung ausländischer Arbeitskräfte. ${ }^{66}$ Zwischen 1960 und 1966 stieg die Zahl der beschäftigten Ausländer von 279 Tsd. auf r,224 Mill.,67 auf die damit der größte Teil der Ausdehnung der Beschäftigung entfiel. ${ }^{65}$

6r Vgl. Soziale Sicherung 1967, Hg. Bundesminister für Arbeit und Sozialordnung, Bonn 1967, S. 122.

62 Vgl. die Übersicht ebda.

63 Zur allgemeinen Entwicklung "aktiver" staatlicher Wirtschaftspolitik vgl. Mückenberger/Welteke, KJ 75, S. Iff.

64 Vgl. Schwarz, in: Geffers/Schwarz, AFG Kommentar, Köln etc. 1974 ff., Ziff. VI, 2

65 Ubersicht in Soziale Sicherung $1967(\mathrm{Vn} .61)$, S. $130 \mathrm{ff}$.

66 Nach $\$$ r der VO vom 18.6. 35 hat die Bundesanstalt das Anwerbungs- und Vermirtlungsmonopol für ausländische Arbeitskräfte.

$67 \mathrm{Vgl}$. den Informationsbericht des IMSF Nr. 19, Ffm 1975, S. 69.

$68 \mathrm{Zu}$ den an diese Situation anknüpfenden Theorien der Ausländerbeschäftigung vgl. Nikolinakos, 
Die Veränderungen des Arbeitsmarkts drückten einen Wandlungsprozeß der OKonomie aus, dem gegenüber die Politik des "CDU-Staats« disfunktional geworden war. Mit dem neoliberalen Wirtschaftskonzept wurde auch sein Sozialstaatsprogramm brüchig, denn Wirtschaftsprozeß und Arbeitsmarkt konnten nicht mehr sich selbst überlassen bleiben. Der Subsidiaritätsgrundsatz, der die Sicherung der sozialen Existenz des Individuums zunächst dem Einzelnen und der Familie, sodann der Sozialversicherung und den Wohlfahrtsverbänden auferlegte, wurde seiner Aufgabe zunehmend weniger gerecht.

Die nur zögernde Anpassung der Arbeitsverwaltung an die veränderten Arbeitsmarktbedingungen führten zu Uberlegungen, die Arbeitsverwaltung, eingebettet in die Forderung nach einer insgesamt veränderten staatlichen Wirtschaftspolitik, grundlegend umzugestalten und die Bildungs- und Mobilitätsförderung in den Mittelpunkt zu stellen. In seinem 2. Gutachten ${ }^{69}$ (1965) wies der Sachverständigenrat zur Begutachtung der gesamtwirtschaftlichen Entwicklung unter Bezugnahme auf amerikanische Erfahrungen auf die Funktion von Qualifikation und Mobilität hin: "Mit dem wachstumsbedingten Strukturwandel der Wirtschaft vollzieht sich ein Wandel im Bedarf an beruflichen Qualifikationen" und "wenn sich der technische Fortschritt beschleunigt, nimmt der Bedarf an Mobilität zu. « 70 (I 57 ) Er diagnostizierte daher die Notwendigkeit der Anpassung und des Ausbaus der Bildungs- und Ausbildungssysteme an den ökonomischen Wandel. Da immer mehr damit zu rechnen sei, "daß Menschen durch die Umstände veranlaßt werden, den ursprünglich erlernten Beruf im Laufe des Berufslebens vielleicht sogar mehrmals zu wechseln" ( $16 \mathrm{I})$, müßten Fortbildungs- und Umschulungsmaßnahmen verstärkt gefördert werden, z. B. durch gesetzliche oder tarifvertraglich begründete Ansprüche auf Bildungsurlaub und die steuerliche Absetzbarkeit der Teilnahme an solchen Maßnahmen..$^{71}$

In seinem nächsten Gutachten ${ }^{72}$ (1966) zog er daraus die institutionelle Konsequenz und schlug vor, die berufliche und regionale Mobilität der Arbeitskräfte durch den Ausbau der Arbeitsverwaltung zu einem Bundesamt für Beschäftigungs- und Strukturpolitik zentral zu fördern und dabei die Umschulungs- und Fortbildungsmaßnahmen auszubauen. ${ }^{73}$

Die Krise 1966/67 verlieh diesen Forderungen Gewicht und führte mit der Beteiligung der SPD an der Regierung der Großen Koalition zu einer Veränderung staatlicher Wirtschafts- und Finanzpolitik, in deren Verlauf die Bereiche staatlicher Aufgaben und Verantwortlichkeiten stark zunahmen.

Im Jahr 1967 stieg die Zahl der Arbeitslosen auf 459 Tsd. (2,1\%), die der Kurzarbeiter auf 543 Tsd. gegenüber 16 Tsd. im Jahr zuvor, während die Zahl der beschäftigten Ausländer um 200 Tsd. zurückging bei einer allgemeinen Abnahme der Beschäftigung um 700 Tsd. Der in den Arbeitsmarktdaten erkennbare zyklische Charakter der Krise wurde überlagert durch Strukturkrisen einzelner Branchen, insbesondere des Bergbaus. ${ }^{74}$

Zentrales Ergebnis der Regierung der Großen Koalition war (neben der Verabschiedung der Notstandsgesetze) die Erweiterung des finanz- und wirtschaftspolitischen

Politische Okonomie der Gastarbeiterfrage, Reinbek 1973, und die Besprechung Neufert, KJ 76, S. 223. 69 BT Drs. V/123.

70 A. a. O., S. 67.

71 A. a. O., S. 161

$72 \mathrm{BT}$ Drs. V/1160.

73 A. a. O., S. 67.

74 Zahlen nach Weller, a. a. O. S. 154, Bergmann u. a., a. a. O., S. 346, IMSF Bericht Nr. 19, S. 63.

Vgl. neben der bereits angeführten Literatur auch Beiträge des IMSF Nr. 4, Wirtschaftskrise und

Wirtschaftspolitik Frankfurt 1976. 
Instrumentariums des Staates. Durch die Eröffnung der Möglichkeit, die Staatshaushalte über Kredite auszugleichen, die verfassungsmäßige Verankerung des "magischen Vierecks" als Maßstab staatlicher Politik und die Einrichtung der Konzertierten Aktion wurden Globalziele und Verfahren produziert, die Staat und Arbeitsmarktparteien auf die Stabilisierung der kapitalistischen Okonomie verpflichteten. Mittels der verfahrensmäßigen Beteiligung der Arbeitsmarktparteien an der Zielfindung staatlicher Politik sollte eine Wirtschafts-, Sozial- und Einkommenspolitik der "sozialen Symmetrie erreicht werden, deren wichtigstes Gebiet der verteilungspolitische Interessenausgleich zwischen Kapital und Lohnarbeit war. Im Globalziel des magischen Vierecks fand der Konsens von Staat und Tarifparteien über den Ausweg aus der Krise seinen Ausdruck, andererseits waren im Keim darin die Auseinandersetzungen um die Priorität der einzelnen Ziele bereits enthalten.

Im AFG und seiner Entstehung drückt sich dieser Konsens exemplarisch aus. Im Juni 1966 forderte der Bundestag die Bundesregierung auf, einen Entwurf zur Novellierung der AVAVG vorzulegen mit dem Ziel, das Gesetz "an den technischen Fortschritt und die wirtschaftliche Entwicklung anzupassen ".7s Im August des gleichen Jahres legte die SPD-Fraktion den Entwurf eines "Arbeitsmarktanpassungsgesetzes « vor $^{76}$, in dessen $\mathbb{I}_{I}$ vier Ziele gesetzt wurden: Förderung der Arbeitsmarkt- und Berufsforschung, Anpassung der Berufsausbildung an die Entwicklung von Wissenschaft und Technik, Förderung der beruflichen Bildung der Arbeitnehmer und die Vermeidung unterwertiger Beschäftigung aus Anlaß von Betriebsänderungen. Er wurde zusammen mit dem AFG-Entwurf der Bundesregierung77 in den Ausschüssen beraten, wobei der Berufsausbildungskomplex abgespalten wurde. ${ }^{78}$

Nach rund zweijähriger Beratung, in deren Verlauf einige Vorwegregelungen durch Änderungen des AVAVG erfolgten, wurde das Gesetz am I 3. 5. 1969 vom Bundestag einstimmig verabschiedet. Dieselbe Einmütigkeit hatte sich bereits in den Beratungen und Anhörungen gezeigt, in denen den Prinzipien des AFG allgemein zugestimmt wurde. ${ }^{79}$

Mit den gegenüber dem AVAVG veränderten Zielen und erweiterten Instrumentarien versucht das AFG eine "zukunftsweisende Arbeits- und Beschäftigungspolitik $\ll^{8 \circ} \mathrm{zu}$ realisieren, indem es die Arbeitsverwaltung in die globale staatliche Wirtschaftspolitik integriert. Nach \$1 AFG sollen die Maßnahmen nach dem AFG dazu beitragen, einen hohen Beschäftigungsstand zu erzielen und aufrechtzuerhalten, die Beschäftigungsstruktur zu verbessern und damit das Wachstum der Wirtschaft zu fördern, wobei nach $\S_{3}$ die Bundesanstalt für Arbeit als Träger der Maßnahmen in die Wirtschafts- und Sozialpolitik der Bundesregierung eingebunden wird.

Dies drückt sich auch in einer verstärkten Position des Staates in der Verwaltung der Bundesanstalt aus. Die Drittelparität gilt nunmehr für alle Belange der Bundesanstalt und nach $\$$ I9I bedürfen alle Anordnungen der Zustimmung zumindest des Bundesministers für Arbeit. Die in den Referentenentwürfen noch vorgesehenen weiteren Einschränkungen der Selbstverwaltung wurden durch energische Proteste der Spitzenorgane der Bundesanstalt und der Tarifparteien bereits im Regierungs-

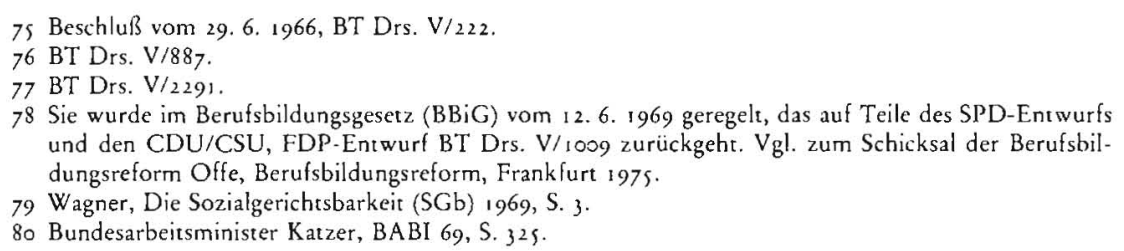


entwurf entfernt. ${ }^{{ }^{1}}$ Wenn auch die Selbstverwaltung formell noch beibehalten ist, stellt Weller zu Recht fest, daß »in Wirklichkeit die Bundesanstalt weitgehend in das BMAS eingegliedert und damit ein Instrument der Exekutive geworden ist ${ }^{82}$

Neben dieser Integration in die allgemeine staatliche Globalsteuerungspolitik formuliert das AFG Ziele, die sich unmittelbar auf die Interessen der von Arbeitslosigkeit betroffenen bzw. bedrohten Arbeitskräfte beziehen. Nach $\ 2$ sollen neben Arbeitslosigkeit auch unterwertige Beschäftigung verhindert, die Mobilität der Arbeitskräfte verbessert und die Eingliederung Behinderter gefördert werden. In den Mittelpunkt der diesem Ziel entsprechenden Maßnahmen stellt das AFG den Gebrauchswert der Arbeitskraft selbst, ihre Qualifikation und den ihn wesentlich mitbestimmenden Faktor, die Mobilität der Arbeitskräfte. Durch Maßnahmen der Förderung der beruflichen Ausbildung und Fortbildung und Mobilitätshilfen soll Arbeitslosigkeit präventiv vermieden werden. Daher geht nach $\S S$ AFG neben der Vermittlung von Arbeit auch die Förderung der beruflichen Bildung der Zahlung von Arbeitslosengeld und Arbeitslosenhilfe vor.

Strukturell benachteiligte Arbeitskräfte, Behinderte und Ältere, werden durch berufliche Rehabilitation und Maßnahmen zur Schaffung von Arbeitsplätzen für ältere Arbeitnehmer gefördert. Daneben wird versucht, einen verbesserten Arbeitsplatzschutz zu erreichen. Die Zahlung von Arbeitslosengeld und Arbeitslosenhilfe soll als letztes Mittel der Sicherung der Existenz bei Arbeitslosigkeit eingreifen. Die für eine präventive Politik erforderlichen Informationen über die Vorgänge am Arbeitsmarkt sollen durch den Ausbau der Arbeitșmarkt- und Berufsforschung beschafft werden $(\$ 6)$.

Der erweiterte Aufgabenbereich der Arbeitsverwaltung drückt zwei sich entsprechende Tendenzen aus. Auf der einen Seite den Vergesellschaftungsprozeß im Bereich der Bildung und Qualifikation: Staatliche Instanzen übernehmen zunehmend die Bereitstellung und Finanzierung der Bildungs- und Qualifikationseinrichtungen und die individuellen Kosten der Ausbildung. Diesem Prozeß korreliert auf Seiten der Arbeitskräfte eine Ausdehnung der Rechtsansprüche auf Qualifikation und deren Finanzierung. ${ }^{83}$

Insofern entwickelt sich die Arbeitsverwaltung zum Keim eines planerischen Systems, das Elemente der zweckmäßigen Verteilung von Qualifikationen enthält. Z. B. macht $\ 36$ die Förderung durch die Bundesanstalt von der arbeitspolitischen Zweckmäßigkeit abhängig, außerdem kann durch die Subventionierung neuer Arbeitsplätze Einfluß auf deren Art und Standort genommen werden. Damit stellt sich das AFG als Realisierung eines Programms dar, das einerseits bestimmten Interessen der Arbeitskräfte an Erhaltung ihrer Arbeitsplätze, an Verbesserung ihrer Qualifikation, an Hilfen für benachteiligte Gruppen, das andererseits die Interessen der Kapitale an adäquat ausgebildeten Arbeitskräften und finanzieller Förderung zusammenfaßt.

In dieser Konzeption reflektieren sich die durch die Krise 1966/67 und ihre Auswirkungen hervorgerufene Einschätzung, die strukturbedingten Krisenprobleme könnten durch entsprechendes "konzertiertes" Verhalten in einem gleichgewichtigen Zustand aufgehoben werden. Auf diesen gleichgewichtigen Zustand einer wachsenden Okonomie sind die Instrumente des AFG bezogen, Mechanismen des Ausgleichs von Angebot und Nachfrage nach Arbeitskräften und der Ausweitung des Beschäftigungspotentials. So werden nach $\mathbb{S} \int_{42}$ a. F. auch bis dahin nicht

8. Schieckel Kommentar zum AFG, S. X.

82 Weller, S. 256 .

$83 \mathrm{Vgl} . \$ \$ 36,40 \mathrm{ff}$. AFG. Zum widersprüchlichen Prozeß der Vergesellschaftung von Sozialisation und Qualifikation vgl. Barabas u. a., Jahrbuch der Sozialarbeit, Reinbek 1975. 
beitragspflichtig Beschäftigte in die Maßnahmen der Arbeitsverwaltung einbezogen.

Zugleich erweist sich das AFG als Moment einer veränderten Sozialstaatspraxis. Bis dahin wurde der Staat im Prinzip als letztes Mittel der Sicherung der sozialen Existenz begriffen, er setzte die private Reproduktion der Individuen voraus und suchte sie durch Nichtintervention aufrechtzuerhalten. Seit der Krise zeigt sich eine neue Praxis, die als Konzeption der "sozialen Förderung " beschrieben werden kann und dem Staat eine aktive Rolle für alle Bereiche der gesellschaftlichen Entwicklung zuweist. Diese setzt zwar weiter an der privaten Reproduktion des Einzelnen an, entwickelt aber für tendenziell alle gesellschaftlichen Bereiche Ziele und Programme, die mittels finanzieller Anreize und Unterstützungen an Private und den Ausbau der vom Staat erbrachten Leistungen realisiert werden sollen und in das Ziel der gesamtwirtschaftlichen Stabilität eingebettet und daraufhin bezogen sind. Durch dieses Uberziehen der Produktion und Reproduktion der Gesellschaft mit finanziellen Vergünstigungen, Subventionen, Darlehen und sonstigen Regelungen zieht der Sozialstaat Verantwortlichkeiten und Kontrollen an sich, die in gleichem Ausmaß die Verantwortung der Privaten reduzieren und damit ihre Existenz zunehmend von staatlichen Regelungen abhängig machen.

Für die Arbeitsverwaltung heißt das, daß durch die vermehrten Regelungen und Finanzmittel, die Arbeitsmarktpolitik und -verwaltung bereitstellen, die Handlungen und Strategien der sich am Arbeitsmarkt Gegenüberstehenden zunehmend abhängig werden von den konkreten Entscheidungen staatlicher Politik, und daß die Arbeitsmarktsituation, Vollbeschäftigung oder Massenarbeitslosigkeit, als Resultat staatlicher Politik erscheinen. ${ }^{84}$

\section{Arbeitsmarktpolitik als Instrument staatlicher Krisenstenerung}

Die dritte Phase des Arbeitsmarktes, die sich an die Krise $1966 / 67$ anschließt, ist gekennzeichnet durch den sich aus der Krise entfaltenden Boom und den anschließenden Abschwung in die Krise 1974/75. Die Arbeitsmarktdaten ${ }^{s_{5}}$ zeigen zunächst eine Ausdehnung des Arbeitsmarkts bis 1973, die Zahl der abhängig Beschäftigten erreicht einen neuen Höchststand, Kurzarbeit und Arbeitslosigkeit gehen zurück, die Zahl der beschäftigten Ausländer nimmt um I,s Mill. zu. Die verbesserten Verwertungsbedingungen nach der "reinigenden " Krise und die günstige Weltmarktposition ermöglichen es dem bundesrepublikanischen Kapital, verstärkt durch staatliche Ankurbelungsmaßnahmen, einen neuen Akkumulationsschub zu vollziehen, der die Wachstumsraten von Investitionen (bis 1970) und Sozialprodukt in die

\begin{tabular}{|c|c|c|c|c|c|}
\hline & $\begin{array}{l}\text { Abhängig } \\
\text { Beschäftigte } \\
\text { insgesamt }\end{array}$ & $\begin{array}{l}\text { davon } \\
\text { Ausländer }\end{array}$ & $\begin{array}{l}\text { Kurz. } \\
\text { arbeiter }\end{array}$ & $\begin{array}{l}\text { Arbeits- } \\
\text { lose }\end{array}$ & $\begin{array}{l}\text { Arbeits - } \\
\text { losen- } \\
\text { quote }\end{array}$ \\
\hline 1967 & 21054 & 1014 & 143 & 459 & 2,1 \\
\hline 1968 & 21183 & 1019 & 10 & 323 & 1,5 \\
\hline 1969 & 21752 & 1366 & 1 & 179 & 0,9 \\
\hline 1970 & 22246 & 1807 & 10 & 149 & 0,7 \\
\hline 1971 & 22414 & 2128 & $\$ 6$ & 185 & 0,9 \\
\hline 1972 & 22435 & 2284 & 76 & 246 & 1,1 \\
\hline 1973 & $22564:$ & 2493 & 44 & 273 & 1,3 \\
\hline 1974 & $22152^{5}$ & 2180 & 292 & 582 & 2,6 \\
\hline 1975 & 21420 & 2061 & 773 & 1074 & 4,7 \\
\hline
\end{tabular}


Höhe schnellen läßt und den Arbeitsmarkt noch einmal ausdehnt. Die Ausdehnung der Beschäftigung vollzieht sich jedoch zunehmend im Dienstleistungsbereich und im öffentlichen Dienst, während sich in der Industrie der Rationalisierungsprozeß der Produktion fortsetzt und die Beschäftigtenzahlen zunächst stagnieren und seit 197r kontinuierlich zurückgehen, ${ }^{\text {S6 }}$ wobei der Export arbeitsintensiver Produktionen in "Niedriglohnländer « als Reaktion auf die erhöhten Lohnzuwachsraten seit Anfang der yoer Jahre eine zunehmende Rolle spielt. Trotz dieser günstigen Entwicklung der Kapitalakkumulation können Kurzarbeit und Arbeitslosigkeit nicht mehr dauerhaft auf das Niveau der 6oer Jahre gedrückt werden.

Auch in den Dienstleistungssektoren und im öffentlichen Dienst setzt die Rationalisierung der Arbeitsprozesse ein, ${ }^{8}{ }^{8}$ sodaß es auch in diesen Bereichen zur Stagnation der Beschäftigung und in der Krise zu "Freisetzungen « kommt. Der Beschäftigungsrückgang in der Krise 1974/75 erfaßt fast alle gesellschaftlichen Arbeitsbereiche und Qualifikationen. ${ }^{88}$

Im Aufschwung schien das AFG tatsächlich im Rahmen der staatlichen Wirtschaftspolitik eine aktive Arbeitsmarktpolitik aus einem Guß zu realisieren. Steigende Einnahmen ermöglichten die reibungslose Finanzierung der sich ausdehnenden Bildungsprogramme und damit die Erfüllung der Aufgabe, die Arbeitsmarktprozesse flexibel auszugleichen. Die Zahl der an den Bildungsmaßnahmen nach dem AFG teilnehmenden Personen stieg von 130 Tsd. im Jahr 1970 auf 270 Tsd. 1975, 200 Tsd. wurden jahresdurchschnittlich in ihrer beruflichen Bildung gefördert; die Ausgaben für diese Maßnahmen stiegen von 770 Mill. DM 1970 auf 2,7 Mrd. DM 1975. Im gleichen Zeitraum stiegen die Gesamtausgaben der Bundesanstalt zur Förderung der Beschäftigung von 990 Mill. DM auf $3,6 \mathrm{Mrd}^{89}$

Während diese Maßnahmen im Prinzip präventiv den Eintritt von Arbeitslosigkeit auf Grund von mangelnder oder unzeitgemäßer Qualifikation verhindern sollen, stieg mit zunehmender Arbeitslosigkeit die Zahl derjenigen, die vor Beginn der Förderung bereits arbeitslos waren. Von 5,8\% (1973) nahm diese Gruppe über $16,3 \%$ (1974) auf $31,3 \%$ (1975) zu, allein unter den Umschülern waren 1975 52,1\% Arbeitslose. ${ }^{\circ}$ In dem Maß, wie die Verwaltung von Arbeitslosigkeit und die Existenzsicherung der Arbeitslosen wieder in den Mittelpunkt der Tätigkeit der Bundesanstalt rückte, veränderte sich der Bezugspunkt der einzelnen Maßnahmen nach dem AFG und trat die präventive Steuerung wieder hinter die reaktive Verwaltung zurück.

In der Praxis der Arbeitsverwaltung kehrt sich damit die Systematik des AFG um.

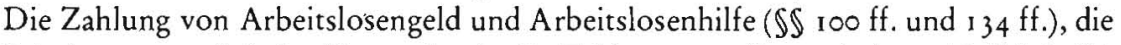
Erhaltung von Arbeitsplätzen durch die Zahlung von Kurzarbeitergeld ( $\$ 6_{3} \mathrm{ff}$.) und die verstärkte Winterbauförderung $(\$ \$ 74 \mathrm{ff}$.), drängen die übrigen Bereiche und Maßnahmen in den Hintergrund und verwandeln deren Charakter. Die Arbeitsbeschaffungsmaßnahmen, die nach $\$ 9$ I dauerhafte Arbeitsplätze schaffen sollen, werden ebenso wie manche Bereiche der beruflichen Bildung tendenziell zur Beschäftigungstherapie,"' während die Maßnahmen zur Förderung der Ausbildung nach $₫ 40$ zunehmend zum Auffangbecken für die aus dem desolaten System der beruflichen Bildung herausgefallenen jugendlichen Arbeitslosen wird. ${ }^{92}$

\footnotetext{
86 Von 8,598 Mill. 1970 auf 7,636 Mill. 1975, nach Monatsberichte der Deutschen Bundesbank 10/76. $87 \mathrm{Vgl}$. dazu Armanski u. a., Staatsdiener im Klassenkampf, Gaiganz 1975 und Haensch, KJ 76, S. iff $88 \mathrm{Vgl}$. für das Personal beim Bund FR vom i ร. 12.1976.

89 Zahlen nach Sozialbericht 1976 und Monatsberichte der Deutschen Bundesbank $10 / 76$.

90 Sozialbericht 1976, S. 23.

91 Zu den Arbeitsbeschaffungsmaßnahmen Finne, links November 1976, S. S.

$92 \mathrm{Vgl}$. dazu Laturner/Schön (Hg.), Jugendarbeitslosigkeit, Reinbek 1975.
} 
Daneben bleibt die klassische Funktion der Arbeitsvermittlung, in die Teile der beruflichen Bildung einzubeziehen sind, auf die Versorgung der gering bleibenden Nachfrage nach Arbeitskräften beschränkt.

Damit erweist sich das AFG in der Krise als im wesentlichen auf die klassischen Bereiche der Arbeitsverwaltung, Arbeitsvermittlung und finanzielle Erhaltung der Arbeitslosen zurückfallendes Gesetz, ein modernisiertes AVAVG, das infolge der Krise und der anhaltenden Massenarbeitslosigkeit selbst in die finanzielle Krise gerät.

Den Hintergrund dieses Rückfalls bildet die Finanzlage der Arbeitsverwaltung in der Krise. Die Finanzierung der Arbeitsverwaltung erfolgt hauptsächlich durch Beiträge, die von Arbeitgebern und Arbeitnehmern je zur Hälfte aufzubringen sind;"3 ihre Höhe ist im Gesetz jeweils festgelegt, sie kann jedoch durch Rechtsverordnung der Bundesregierung nach Lage des Arbeitsmarktes und der Finanzen der Bundesanstalt zeitweilig gesenkt werden $(\$ \$ 167,174, I 1)$. Der Beitragssatz wurde von $6,5 \%$ I 949 bis auf zeitweise $0,0 \% 1961 / 62$ gesenkt und stieg dann kontinuierlich auf $2 \% .{ }^{94}$

Die Kosten der produktiven Winterbauförderung und des Konkursausfallgeldes werden durch Umlagen aufgebracht ( $\$ \$$ I $86 \mathrm{aff}$.), während die Arbeitslosenhilfe im Prinzip nach Art. I 20 GG vom Bund zu tragen ist. $\$ 240$ hat jedoch die Regelung aus den 6oer Jahren beibehalten, nach der die "Anschlußarbeitslosenhilfe" (Arbeitslosenhilfe im Anschluß an Arbeitslosengeld) von der Bundesanstalt finanziert wird. Dieser Finanzierungsmodus konnte bei steigenden Beschäftigungszahlen und steigenden Löhnen die zunehmenden Aufgaben der Bundesanstalt problemlos finanzieren. 1974 und 1975 stiegen jedoch die Ausgaben für Arbeitslosengeld und Anschlußarbeitslosenhilfe auf 4,452 bzw. 10,749 Mrd. DM, was trotz Abbau der Rücklagen der Bundesanstalt Bundeszuschüsse nach Art. I $20 \mathrm{GG}$ von über s Mrd. DM nötig machte.95

Da die rückläufige Beschäftigung auch im Staatshaushalt Einnahmeausfälle verursachte, wurde eine finanzielle Sanierung der Arbeitsverwaltung notwendig, eine Situation, die strukturell, wenn auch nicht in den Ausmaßen, derjenigen Ende der 2oer Jahre glich und mit den gleichen Mitteln in Angriff genommen wurde: mit Leistungsreduzierungen und einer Beitragserhöhung.

Mit dem Haushaltsstrukturgesetz vom I 8. 12. I975 wurde der Beitrag zur Arbeitslosenversicherung auf $3 \%$ erhöht. Die Voraussetzungen des Anspruchs auf Förderung der beruflichen Bildung wurden verschärft, ${ }^{96}$ während das Unterhaltsgeld reduziert wurde und teilweise nurmehr als Darlehen vergeben wird. Die Förderung von vorher nicht beitragspflichtig beschäftigten Personen wurde auf Ausnahmefälle beschränkt. ${ }^{96}$

Wichtigste Änderung des AFG ist jedoch die Verschärfung der Zumutbarkeitsregelung in $\ 103,{ }^{97}$ womit das Ziel des AFG, unterwertige Beschäftigung zu verhindern, aufgegeben wird. Mit der Bestimmung, daß nunmehr auch eine Arbeit zumutbar ist, die nicht mehr der Qualifikation des Arbeitslosen entspricht, geringer entlohnt wird und weiter entfernt ist als bisher, wird es zusätzlich zu den aus der Krise resultierenden lohnmindernden Tendenzen möglich, quasi von Amts wegen die Dequalifika-

Die nach $\$ 57$ subsidiäre berufliche Rehabilitarion und das Konkursausfallgeld nach $\$ 14$ Ia AFG bleiben hier außer Betracht.

93 Die Beitragspflicht ist immer mehr ausgedehnt worden, Ausnahmen regeln die $\$ \$ 169 \mathrm{ff}$. AFG.

94 Eine genaue Ubersicht in Soziale Sicherung 1975 (Hg. BMAS), S. 231.

95 Zahlen nach Monatsberichte, a. a. O. und Sozialbericht 1976, S. 143.

96 Die konkrete Förderungspraxis beschreibt der Frankfurter Arbeitsamtsdirektor in der FR vom is. I. 1977 so: "Was arbeitsmarktpolitisch zweckmäßig ist, bestimme ich." 97 Dazu bereits Wacker/Paul (Fn. 2). 
tion eines Arbeitslosen und die Reduktion seines Einkommens durchzusetzen, da die Verweigerung der Vermittlung zur Sperrung der Arbeitslosenunterstützung führt..$^{98}$

Die Sanierung der Finanzen der Bundesanstalt erfolgt auf Kosten der Lohnabhängigen und der Arbeitslosen. Die Deutsche Bundesbank berichtet, daß die Ausgaben für berufliche Bildungsmaßnahmen bereits um $25 \%$ zurückgegangen sind, "da die Zahl der Bezieher des bei Fortbildungs- und Umschulungskursen gewährten Unterhaltsgeldes bereits erheblich zurückgegangen ist «. .9 Hinzu kommt die Aussteuerung einer zunehmenden Zahl von längerfristig Arbeitslosen aus dem Bezug von Arbeitslosengeld, unter denen ein weiterer Teil durch die Bedürftigkeitsprüfung aus dem Bezug von Arbeitslosenhilfe ausgeschlossen wird.

Dieser Prozeß erinnert an die Sanierung der Reichsanstalt für Arbeitsvermittlung und Arbeitslosenversicherung. Nach dem Bruch der Großen Koalition 1930, Resultat der Auseinandersetzungen um die Finanzierung der Arbeitslosenversicherung, ${ }^{100}$ wurden durch Beitragserhöhungen, Aussteuerungen und Leistungsreduzierungen die Finanzen der Reichsanstalt soweit saniert, daß sie 1932 steigende Úberschüsse aufwies; bei 5,15 Mill. gezählten Arbeitslosen wurden allerdings auch nur noch 0,618 Mill. unterstützt. ${ }^{101}$

Angesichts eines wachsenden Arbeitskräftepotentials in Gestalt der auf den Arbeitsmarkt zukommenden geburtenstarken Jahrgänge einerseits und anhaltender Rationalisierungsprozesse in allen Arbeitsbereichen andererseits, dürften mit den bisherigen Maßnahmen die Finanzierungsprobleme nicht beseitigt sein. Absehbar ist auch nicht, wie mit dem aktuellen Instrumentarium des AFG angesichts der ökonomisch restriktiven Politik des Staates eine Ausdehnung der Beschäftigung erfolgen soll.

\footnotetext{
98 Detailliert zu den einzelnen Änderungen Siegers, BABI. 76, S. 46. 99 Monatsberichte der Deutschen Bundesbank 9/76, S. 21.

10 Vgl. Timm (Fn. 42).

$10 \mathrm{r}$ Vgl. Preller, a. a. O., S. $418 \mathrm{ff}$., insbes. $448 \mathrm{ff}$.
} 